\title{
Weak-force sensing with squeezed optomechanics
}

\author{
Wen Zhao, ${ }^{1,2, *}$ Sheng-Dian Zhang, ${ }^{1, *}$ Adam Miranowicz, ${ }^{3}$ and Hui Jing ${ }^{1, \dagger}$ \\ ${ }^{1}$ Key Laboratory of Low-Dimensional Quantum Structures and Quantum Control of Ministry of Education, \\ Department of Physics and Synergetic Innovation Center for Quantum Effects and Applications, \\ Hunan Normal University, Changsha 410081, China \\ ${ }^{2}$ College of Physics and Materials Science, Henan Normal University, Xinxiang 453007, China \\ ${ }^{3}$ Faculty of Physics, Adam Mickiewicz University, 61-614 Poznań, Poland
}

(Dated: September 2, 2019)

\begin{abstract}
We investigate quantum-squeezing-enhanced weak-force sensing via a nonlinear optomechanical resonator containing a movable mechanical mirror and an optical parametric amplifier (OPA). Herein, we determined that tuning the OPA parameters can considerably suppress quantum noise and substantially enhance force sensitivity, enabling the device to extensively surpass the standard quantum limit. This indicates that under realistic experimental conditions, we can achieve ultrahighprecision quantum force sensing by harnessing nonlinear optomechanical devices.
\end{abstract}

\section{INTRODUCTION}

Cavity optomechanics (COM) has recently emerged as a versatile platform for both fundamental studies of lightmatter interactions and practical applications ranging from optical communication to quantum metrology $[1,2]$. In particular, COM sensors have achieved unprecedented sensitivity for measuring mass [3, 4], acceleration [5, 6], displacement [7-10], and force [11-16]. Moreover, the sensitivity of such sensors is constrained by the standard quantum limit (SQL) [17] or a lower bound on the additional measurement uncertainty determined by the balance between the shot and back-action noise. However, the SQL has been surpassed using quantum non-demolition techniques [18-20] to achieve sub-SQL sensitivity via quantum entanglement [21-23] or squeezing [24-31]. Squeezed states were studied early in 1927 [32], although an explosion of interest in them was triggered 40 years ago when they were first used to detect gravitational waves via supersensitive interferometry [11, 24, 33, 34]. Experiments by injecting squeezed light into a COM resonator have successfully demonstrated sub-SQL sensitivity [35-38]. However, the inevitable injection losses hinder the ultimate performance of COM sensors in practice.

To overcome this obstacle, COM sensing using an intracavity optical parametric amplifier (OPA) has recently been proposed to implement ultrahigh-precision position detection [39]. This scheme has the advantage that all the information is imprinted on the deamplified momentum quadrature, which induces limited signal suppression but simultaneously brings about a dramatic reduction in the noise. Using such squeezed resonators, the SQL can be attained precisely at a mechanical resonance without injection losses [39]. Additionally, sub-SQL sensitivity can be achieved using an OPA in a dissipative COM system [40]. Nevertheless, none of these

\footnotetext{
* These authors contributed equally to this work.

$\dagger$ jinghui73@foxmail.com
}

previous works have considered the role of the optical phase, particularly the OPA pump phase, in further enhancing the sensitivity.

This paper aims to fill this gap by discussing the effects of both the OPA gain and pump phase on force sensing in a squeezed COM device. We show that tuning the OPA parameters can considerably suppress quantum backaction noise and enable the device to reach sub-SQL sensitivity at a smaller COM coupling without losing quantum efficiency. Unlike previous studies [39], our study focuses on the squeezed quadrature and we determine that in the limit where the cavity linewidth is much larger than any measurement frequency of interest [41], the squeezed momentum quadrature carries complete information regarding the weak-force signals, and the information about the added force contained in the position quadrature can be safely ignored. Herein, we focus on canonical quadrature squeezing; however, other observables can also be squeezed, such as the photon number $[42,43]$. Our research demonstrates that squeezed COM devices are feasible and powerful enough to achieve ultrahigh precision quantum measurement [44, 45].

\section{MODEL AND SOLUTION}

Figure 1 shows the schematics of intracavity squeezing in an OPA-assisted Fabry-Pérot cavity [46, 47]. Previous researches on such squeezed quantum systems have usually focused on enhanced mechanical cooling [4851] or squeezing [52-54] and enhanced light-matter coupling [55-57]. When light pressure couples to a movable mirror, coherent states are transformed into squeezed states of light [58], and this type of squeezing, referred to as ponderomotive squeezing [59], can be only used to evade back-action and thus it is less extensive than externally injecting a squeezed light [60] or generating intracavity squeezing via an OPA [46]. We note that in a recent experiment, the OPA effect was improved by 14 orders of magnitude via symmetry breaking at a microcavity surface [61]. Other approaches 


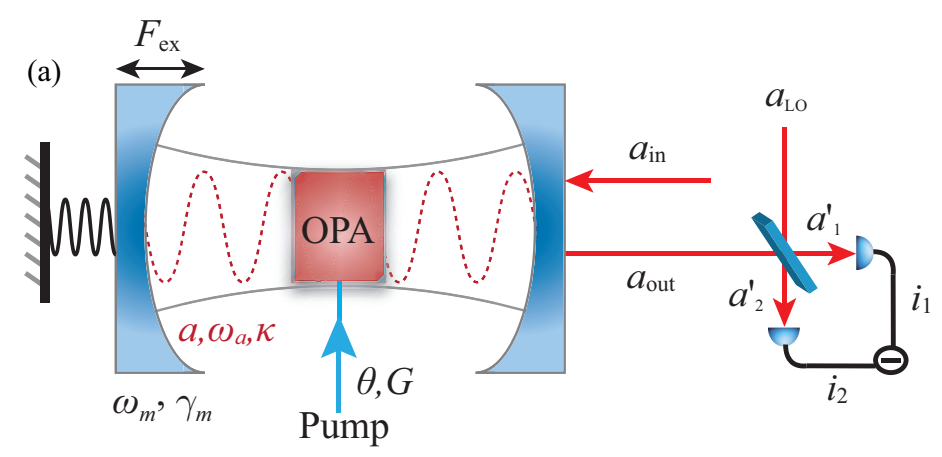

(b)
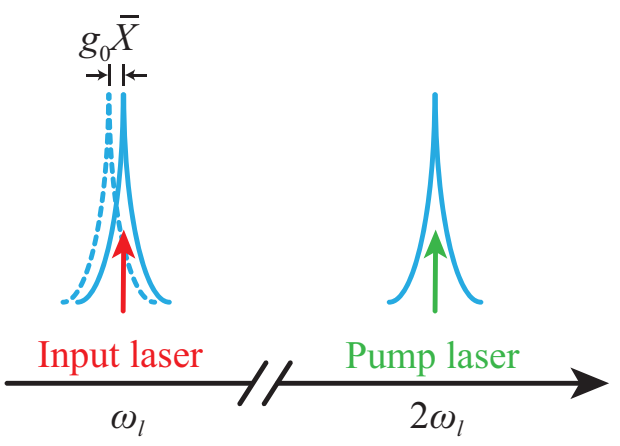

FIG. 1. (Color online) (a) Schematics of the cavity optomechanical (COM) system consisting of a Fabry-Pérot cavity coupled to a degenerate optical parametric amplifier (OPA). One or even two mirror coatings are usually directly placed on the spherical and polished surfaces of the nonlinear crystal that generates the squeezed vacuum and produces parametric amplification [46, 47]. In this setup, an external weak force $F_{\text {ex }}$ acting on the mechanical resonator can be measured with homodyne detection. (b) Frequency spectrum of the OPA-assisted COM system. An input laser drives the optical cavity at frequency $\omega_{l}$, thus, a pump laser beam with frequency $2 \omega_{l}$ is applied to the degenerate OPA. The dashed curve shows the static frequency shift $g_{0} \bar{X}$ at the cavity resonance because of radiation pressure.

to generate intracavity squeezing include, e.g., Kerr media [62-64] or dissipative COM devices [65-67]. We also note that by using mechanical squeezing [68-71], the sensitivity of detecting small displacements was improved by a factor of up to 7 [70].

To realize weak-force sensing with OPA, we assume that only one optical mode is coupled to the mechanical mode. Thus, the Hamiltonian of the COM system can be described as [53]:

$$
\begin{aligned}
H_{0}= & \hbar \omega_{a} a^{\dagger} a+\frac{p^{2}}{2 m}+\frac{1}{2} m \omega_{m}^{2} x^{2}+\hbar \frac{\omega_{a}}{L} x a^{\dagger} a \\
& +i \hbar G\left(e^{i \theta} a^{\dagger 2} e^{-2 i \omega_{l} t}-e^{-i \theta} a^{2} e^{2 i \omega_{l} t}\right)
\end{aligned}
$$

where $\omega_{a}$ denotes the optical resonant frequency; $x$ and $p$ refer to the position and momentum operators of the vibrating mechanical oscillator having an effective mass $m$ and an angular frequency $\omega_{m}$, respectively. In addition, $a$ and $a^{\dagger}$ are the annihilation and creation operators of the cavity mode, respectively. We have furthermore used $G$ to denote the nonlinear gain of degenerate OPA with $\theta$ being the phase of the pump field driving the OPA medium. For the sake of simplicity, we define dimensionless position and momentum operators of the mechanical mode as $X=x / x_{\mathrm{zpf}}, P=p / p_{\mathrm{zpf}}$, where $x_{\mathrm{zpf}}$ and $p_{\mathrm{zpf}}$ are the standard deviations of the zero-point motion and momentum of the oscillator, respectively, $x_{\mathrm{zpf}}=\sqrt{\hbar /\left(m \omega_{m}\right)}, p_{\mathrm{zpf}}=\sqrt{\hbar m \omega_{m}}$, and the operators $X$ and $P$ satisfy the commutation relation $[X, P]=i$. Thus, in a rotating frame, the Hamiltonian can be rewritten as follows:

$$
\begin{aligned}
H= & \hbar \Delta_{a} a^{\dagger} a+\frac{\hbar}{2} \omega_{m}\left(X^{2}+P^{2}\right)+\hbar g_{0} a^{\dagger} a X \\
& +i \hbar G\left(e^{i \theta} a^{\dagger 2}-e^{-i \theta} a^{2}\right),
\end{aligned}
$$

where $g_{0}=x_{\mathrm{zpf}} \omega_{a} / L$ represents the single photon coupling strength of the COM interaction. The first and second terms in Eq. (2) denote the sum of the free energy of the cavity field and the mechanical mode without external forces, respectively. And the last two terms respectively describe the COM interaction and the contribution of an OPA. By introducing dissipation and noise terms, the Heisenberg-Langevin equations can be written as:

$$
\begin{aligned}
\dot{X} & =\omega_{m} P, \\
\dot{P} & =-\omega_{m} X-\gamma_{m} P-g_{0} a^{\dagger} a+\sqrt{2 \gamma_{m}}\left(f_{\mathrm{th}}+f_{\mathrm{ex}}\right), \\
\dot{a} & =-\left(i \Delta_{a}+\frac{\kappa}{2}\right) a-i g_{0} X a+2 G e^{i \theta} a^{\dagger}+\sqrt{\kappa} a_{\mathrm{in}},
\end{aligned}
$$

where $\Delta_{a}=\omega_{a}-\omega_{l}$ is the detuning of the input light frequency $\left(\omega_{a}\right)$ with respect to the cavity resonant frequency $\left(\omega_{l}\right) ; a_{\text {in }}$ characterizes the input field driving the cavity, which fulfils [41, 49]: $\left\langle a_{\mathrm{in}}(t) a_{\mathrm{in}}^{\dagger}\left(t^{\prime}\right)\right\rangle=$ $\delta\left(t-t^{\prime}\right)$. Moreover, $f_{\mathrm{th}}=\xi / \sqrt{2 \hbar m \gamma_{m} \omega_{m}}$ and $f_{\mathrm{ex}}=$ $F_{\text {ex }} / \sqrt{2 \hbar m \gamma_{m} \omega_{m}}$ are the scaled thermal and external forces with zero mean values, respectively. The Brownian thermal noise operator $\xi$ obeys the correlation function [72]:

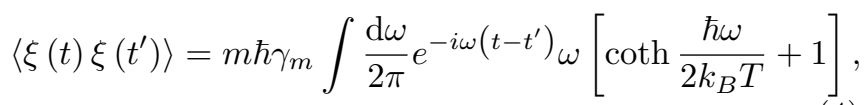

where $k_{B}$ is the Boltzmann constant and $T$ is the mirror temperature of the thermal bath. Additionally, if the mechanical quality factor is large, $Q=\omega_{m} / \gamma_{m} \gg 1$, the Brownian noise $\xi(t)$ describes a Markovian process that is delta-correlated [72]:

$$
\frac{1}{2}\left\langle\xi(t) \xi\left(t^{\prime}\right)+\xi\left(t^{\prime}\right) \xi(t)\right\rangle \simeq m \hbar \omega_{m} \gamma_{m}(2 \bar{n}+1) \delta\left(t-t^{\prime}\right),
$$

where $\bar{n}=\left[\exp \left(\hbar \omega_{m} / k_{B} T\right)-1\right]^{-1}$ is the mean thermal phonon number. Under the approximation of thermal equilibrium and taking the classical limit 
$\hbar \omega_{m} \ll k_{B} T$ [17], the scaled thermal force obeys $\left\langle f_{\text {th }}(t) f_{\text {th }}\left(t^{\prime}\right)\right\rangle=\left(k_{B} T / \hbar \omega_{m}\right) \delta\left(t-t^{\prime}\right)[41]$.

Linearization or even semiclassical approximation are standard effective descriptions applied in case of strong optical drives, which are valid in both theory [26, 40] and experiments [15, 73, 74]. Indeed, a strong quantumoptical drive can usually be treated as a semiclassical parameter. Thus, an operator (a q-number) describing such a drive can be replaced by a c-number. For example, in standard description of homodyne detection, which can be applied for quantum state tomography, a weak quantum signal is driven (i.e., mixed on a beam splitter) by a strong-laser mode (i.e., a local oscillator), which is described by a classical parameter (see Appendix D). Therefore, in case where the input optical field is a semiclassical coherent laser field [15], i.e., in the strong driving regime $|\alpha| \gg 1$ [40] (see Appendix B), we can expand each operator as the sum of its steady-state value and a small fluctuation, i.e., $P=\bar{P}+\delta P, X=\bar{X}+\delta X$, $a=\alpha+\delta a$, and $a_{\text {in }}=\alpha_{\text {in }}+\delta a_{\text {in }}$. Here we choose the input field as the zero-phase reference, i.e., $\alpha_{\text {in }}=\left|\alpha_{\text {in }}\right|=$ $\sqrt{P_{\text {in }} / \hbar \omega_{l}}$ with $P_{\text {in }}$ being the input laser power [17]. By setting all the time derivatives to zero, the steadystate values of the dynamical variables can be obtained as $\bar{P}=0, \bar{X}=-g_{0} \alpha^{*} \alpha / \omega_{m}$ and

$$
\alpha=\frac{\sqrt{\kappa} \alpha_{\text {in }}}{2 \sigma_{+}}\left(\kappa-2 i \Delta+4 G e^{i \theta}\right)=|\alpha| e^{i \phi},
$$

where $\sigma_{ \pm}=\kappa^{2} / 4 \pm \Delta^{2} \mp 4 G^{2}$ and $\Delta=\Delta_{a}+g_{0} \bar{X}$ denotes the effective cavity detuning. Therefore, the phase of the intracavity amplitude becomes:

$$
\phi=\arctan \left(\frac{4 G \sin \theta-2 \Delta}{4 G \cos \theta+\kappa}\right) .
$$

We define the standard quadratures of the cavity field as $x_{a}=\left(a+a^{\dagger}\right) / \sqrt{2}, p_{a}=\left(a-a^{\dagger}\right) /(\sqrt{2} i)$. These are the canonical (i.e., dimensionless) position $\left(x_{a}\right)$ and momentum $\left(p_{a}\right)$ operators, also referred to as the amplitude and phase quadratures, which are related to the electric and magnetic fields of an optical mode, respectively. For simplicity, we set the integral constants to zero and neglect the higher-order terms $\delta a^{\dagger} \delta a$ and $\delta X \delta a$, then the linearized equations can be written as (a similar matrix form is given in Ref. [53]):

$$
\dot{\mathrm{v}}=\mathrm{Cv}+\mathrm{Av}_{\mathrm{in}} .
$$

The operator vectors are $\mathrm{v}=\left(X, P, x_{a}, p_{a}\right)^{\mathrm{T}}$ and $\mathrm{v}_{\text {in }}=\left(0, f_{\text {in }}, x_{a}^{\text {in }}, p_{a}^{\text {in }}\right)^{\mathrm{T}}$, where $f_{\text {in }}=f_{\text {th }}+f_{\text {ex }}$, and superscript $\mathrm{T}$ represents the transpose of a matrix. The quadratures of the input field $\left(x_{a}^{\mathrm{in}}\right.$ and $\left.p_{a}^{\mathrm{in}}\right)$, are defined analogously to $x_{a}$ and $p_{a}$. The coefficient matrix $\mathrm{C}$ and the noise matrix A are given in Appendix B.

In standard homodyne detection, which enables quantum state tomography, a quantum optical field [in our case $\left.a_{\text {out }}(t)\right]$ is mixed with a strong classical laser field $\alpha_{\mathrm{LO}}$ (i.e., a local oscillator, LO) at a balanced beam splitter. The homodyne signal (say, the photocurrent $\left.i_{\mathrm{det}}\right)$ corresponds to the difference of the photon numbers at the two output ports of the beam splitter. This signal is proportional to the generalized phase-dependent quadrature operator $x_{a, \text { out }}^{\varphi}$, as follows [75]:

$$
\begin{aligned}
i_{\mathrm{det}} & =\left|\alpha_{\mathrm{LO}}\right|\left(e^{i \varphi} a_{\mathrm{out}}^{\dagger}+e^{-i \varphi} a_{\mathrm{out}}\right) \\
& =\sqrt{2}\left|\alpha_{\mathrm{LO}}\right| x_{a, \text { out }}^{\varphi},
\end{aligned}
$$

where $\varphi$ and $\alpha_{\mathrm{LO}}$ respectively correspond to the phase and amplitude of the local oscillator, which are arbitrary (see Appendix D for more details). Note that by changing the phase $\varphi$ of the local oscillator, a complete quantum state tomography can be implemented with this method [75]. As depicted in Figure 1, homodyne detection is a phase-referenced technique, where direct measurement of an optical field produces a stochastic photocurrent that is proportional to the rotated quadrature $x_{\mathrm{a} \text {,out }}^{\varphi}$ and the measured quadrature is dependent on the phase of the local oscillator $\alpha_{\mathrm{LO}}$. We also note that the quadratures for arbitrary phases of the local oscillator can be measured. Thus, homodyne tomography can be performed to reconstruct the output field with arbitrary phase [76]. However, for brevity, we further study only the output canonical momentum quadrature, i.e., $\varphi=\pi / 2$. Hence, the external force can be expressed as:

$$
F=\frac{1}{F_{f}(\omega)} x_{\mathrm{a}, \text { out }}^{\pi / 2}=\frac{1}{F_{f}(\omega)} \tilde{p}_{a}^{\text {out }}=\tilde{f}_{\mathrm{ex}}+\tilde{f}_{\mathrm{add}} .
$$

where $F_{f}(\omega)=g f_{-} \chi_{-} \chi_{m} \sqrt{2 \kappa \gamma_{m}}$, and $g=\sqrt{2} g_{0}|\alpha|$ represents the effective linearized optomechanical coupling rate. Therefore, noise of the added force can be described by

$$
f_{\text {add }}(\omega)=\tilde{f}_{\text {th }}+X_{a}(\omega) \tilde{x}_{a}^{\text {in }}+P_{a}(\omega) \tilde{p}_{a}^{\text {in }},
$$

where $\tilde{f}_{r} \equiv f_{r}(\omega), \tilde{s}_{a}^{u} \equiv s_{a}^{u}(\omega)$, for $s=x, p, u=$ in, out, and $r=$ in, th, ex, add. Moreover, the coefficients in Eq. (11) are given by:

$$
X_{a}(\omega)=\left(\mu_{+} \lambda_{+} \chi_{-} \kappa\right) / F_{f}, \quad P_{a}(\omega)=\left(\chi_{-} \kappa-1\right) / F_{f} .
$$

The parameters $f_{ \pm}, \chi_{ \pm}, \mu_{ \pm}$, and $\lambda_{ \pm}$are defined in Appendix C.

We use the symmetric part $\left(S_{\mathrm{FF}}\right)$ of the added noise power spectral density $\left(S_{\mathrm{F}}\right)$ to characterize the sensitivity of the force measurement, given by (see Appendix D for more details):

$$
\begin{aligned}
S_{\mathrm{FF}}(\omega) & =\frac{S_{\mathrm{F}}(\omega)+S_{\mathrm{F}}(-\omega)}{2} \\
& =\underbrace{\frac{k_{B} T}{\hbar \omega_{m}}}_{\text {thermal noise }}+\underbrace{\frac{1}{2}\left|X_{a}(\omega)\right|^{2}}_{\text {backaction noise }}+\underbrace{\frac{1}{2}\left|P_{a}(\omega)\right|^{2}}_{\text {shot noise }},
\end{aligned}
$$


where we have used the correlation functions and the bath cross-correlated terms of the measured symmetrized power spectral density are cancelled out. The first term of $S_{\mathrm{FF}}(\omega)$ represents the thermal Brownian noise. The second term is the back-action noise, which is proportional to the input power $P_{\text {in }}$ and the square of the coupling strength $g^{2}$. Very recently, Corbitt et al. have presented a testbed for the broadband measurement of quantum back action at room temperature [77]. The third term denotes the shot noise that is inversely proportional to the input power $P_{\text {in }}$. Since beating the SQL in an optomechanical sensor by cavity detuning has been studied in Ref. [78] and the highest parametric gain is achieved at the cavity resonance [59], in the following, we neglect thermal noise and other technical noises and restrict our discussion to the case of $\Delta=0$. We note that the idea of utilizing a nondegenerate OPA-assisted COM to circumvent measured backaction and surpass the SQL has been proposed in Ref. [41]. This scheme is based on an antinoise process (using an oscillator with an effective negative mass) via destructive quantum interference, i.e., the so-called coherent quantum noise cancellation (CQNC) [16, 79, 80]. Additionally, a quantummechanics-free subsystem (QMFS) [80], proposed by Tsang and Caves [79], was first realized in Ref. [81].

In order to compare force sensing in the presence and in the absence of OPA, we first concern about force sensing of a standard COM system at a resonant frequency. For such a scheme, the sensitivity cannot surpass the SQL without an OPA [see Figure 2(a)]. Moreover, if we assume the linewidth of the cavity to be much larger than any measurement frequency of interest, $\kappa \gg \omega$, the symmetrized noise spectral density can be simplified as follows [26, 41]:

$$
S_{\mathrm{FF}}^{\mathrm{st}}=\frac{g^{2}}{\kappa \gamma_{m}}+\frac{1}{16} \frac{\kappa}{g^{2} \gamma_{m}} \frac{1}{\left|\chi_{m}\right|^{2}},
$$

where the susceptibility of the mechanical oscillator $\left(\chi_{m}\right)$ has been defined in Appendix B. Thus, we obtain $S_{\mathrm{FF}}^{\mathrm{st}} \geq$ $\left(2 \gamma_{m}\left|\chi_{m}\right|\right)^{-1}=S_{\mathrm{SQL}}, g_{\mathrm{SQL}}^{2}=\kappa /\left(4\left|\chi_{m}\right|\right)$.

Subsequently, we study the case of the zero pump phase $(\theta=0)$ of the COM system with an OPA. In this case, the symmetrized noise power spectral density $S_{\mathrm{FF}}$ can be reduced to:

$$
S_{\mathrm{FF}}(\theta=0)=\frac{g^{2} \kappa}{4 \gamma_{m}(\kappa / 2-2 G)^{2}}+\frac{(\kappa / 2-2 G)^{2}}{4 \kappa \gamma_{m} g^{2}\left|\chi_{m}\right|^{2}} .
$$

Therefore, we also have $S_{\mathrm{FF}}(\theta=0) \geq 1 /\left(2 \gamma_{m}\left|\chi_{m}\right|\right)=$ $S_{\mathrm{SQL}}$, indicating that the detection sensitivity still cannot surpass the SQL when $\theta=0$. As Figure 2(a) shows, in the absence of OPA $(G / \kappa=0)$, the minimum of $S_{\mathrm{FF}} / S_{\mathrm{SQL}}$ equals to 1 when the coupling strength is $g=g_{\mathrm{SQL}}$. The optimal COM parameter $g_{\text {opt }}$ can be obtained by solving $\left|X_{a}\right|=\left|P_{a}\right|$, which gives $g_{\mathrm{opt}}^{(\theta=0)}=$ $|\kappa-4 G| /\left(2 \sqrt{\kappa\left|\chi_{m}\right|}\right)$. Furthermore, by the inspection of Eq. (7) under the resonance condition $\Delta=0$, it is easy (a)

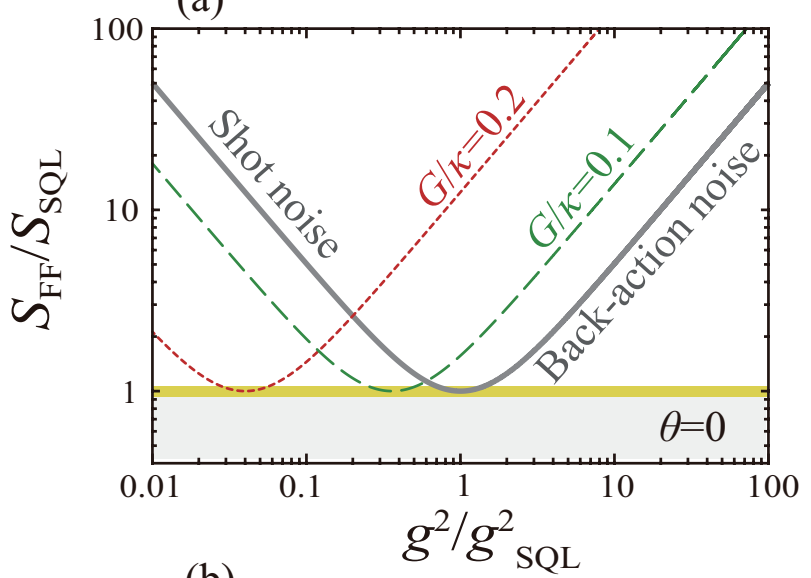

(b)

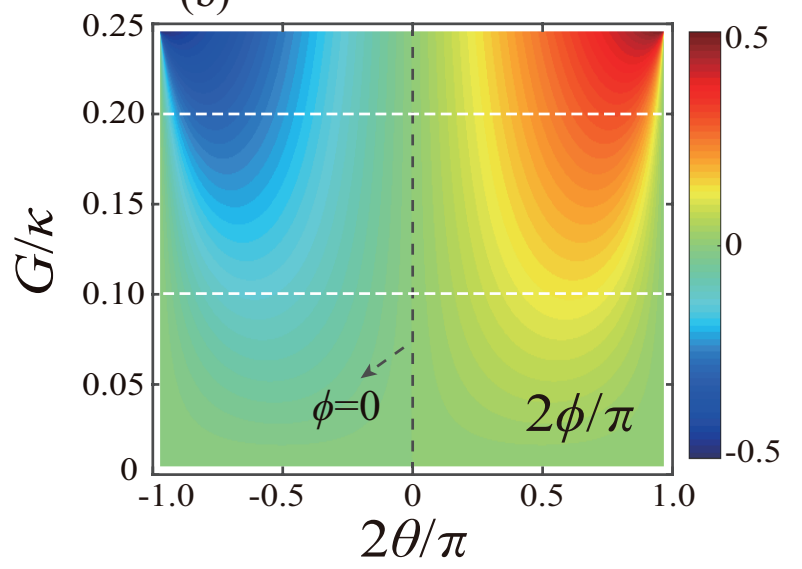

FIG. 2. (Color online) (a) Noise power spectral density $S_{\mathrm{FF}} / S_{\mathrm{SQL}}$ is plotted as a function of the scaled square of the coupling strength $g^{2} / g_{\mathrm{SQL}}^{2}$ and of the OPA parametric gain $G$. The gray solid line corresponds to the non-OPA case. (b) Phase $\phi$, given in Eq. (7), of the intracavity field versus the OPA parameters: the phase $\theta$ and gain $G$ in the units of the damping rate $\kappa$. Here we assume the resonance condition $\Delta=0$.

to understand why the intracavity field phases $\phi$ for $\pm \theta$, corresponding to symmetric points on both sides of the dividing dashed line in Figure 2(b), have opposite signs. By comparing the phases $\phi$ for $\theta= \pm \pi / 2$ and $G / \kappa=1 / 4$, corresponding to the darkest blue and darkest red points in Figure 2(b), we find that the intracavity field phases $\phi$ are $\pi / 2$-shifted for the opposite phases $\theta$ of the OPA pump for these special values of $G=\kappa / 4$ and $\Delta=0$. This conclusion can easily be drawn by analyzing Eq. (7) for the discussed parameters.

From the analyses made above, we have identified that it is impossible for weak-force sensing to exceed the SQL with non-OPA or $\theta=0$. As depicted in Figure 2(a), owing to the reduction of shot noise, $S_{\mathrm{FF}}$ first decreases with the COM coupling strength increasing until the turning point (corresponding to $S_{\mathrm{FF}}=S_{\mathrm{SQL}}$ ); then, the backaction noise is dominant, leading $S_{\mathrm{FF}}$ to increase. Hence, the SQL can be reached at the 
(a)

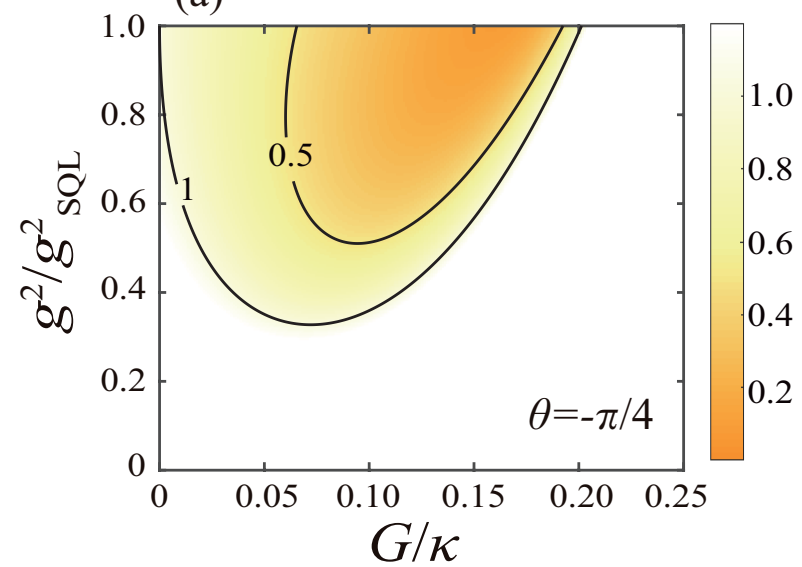

(b)

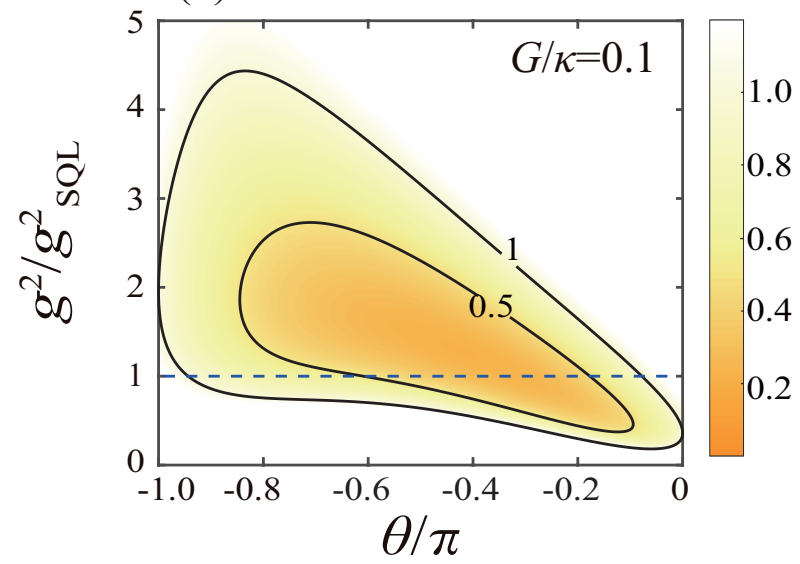

FIG. 3. (Color online) Noise power spectral density $S_{\mathrm{FF}} / S_{\mathrm{SQL}}$ as the functions of the scaled square of the coupling strength, $g^{2} / g_{\mathrm{SQL}}^{2}$, and the parametric gain $G$ or the parametric phase $\theta$. The other parameters are set as (a) $\theta=-\pi / 4$ and (b) $G / \kappa=0.1$.

minimum point $g=g_{\text {opt }}$ and the optimum coupling strength can be lowered by adjusting the OPA pump gain $G$. In calculations, we use experimentally accessible parameters [17], i.e., $\omega_{l} / 2 \pi=2 \times 10^{14} \mathrm{~Hz}, \gamma_{m} / 2 \pi=$ $1 \mathrm{kHz}, \omega_{m} / 2 \pi=10 \mathrm{MHz}, \kappa / 2 \pi=10 \mathrm{MHz}, g_{0} / 2 \pi=$ $100 \mathrm{~Hz}$ and $P_{\text {in }}=700 \mathrm{nW}$.

To overcome the SQL, we study the impact of the OPA pump phase on weak-force sensing. Figure 3 shows the sensitivity as the functions of $g^{2} / g_{\mathrm{SQL}}^{2}$ and the parametric gain $G$ in the low-frequency domain. Specifically, as depicted in Figure 3(a), the sensitivity can be improved more than twice with an OPA when choosing appropriate parameters and the coupling strength required to increase the sensitivity becomes much smaller than $g_{\mathrm{SQL}}$. Additionally, according to Eqs. (12) and (13), we are likely to enhance the detection sensitivity by tuning $\sin \theta$ to satisfy $2 G \sin \theta+g^{2} \chi_{m} \cos ^{2} \phi \approx 0$, for suppressing the backaction noise to the limit. Furthermore, we utilize $\sin \theta<0$ in Figure $3(\mathrm{~b})$ to reduce quantum noise and improve the measurement accuracy. We note that very recently, optimal cavity squeezing up to $30 \mathrm{~dB}$ has been predicted theoretically [82], and single labelfree sensing of nanoparticles has been studied with a spinning resonator [83] and exposed-core fiber [84]. In particular, Miao et al. have recently proposed a new fundamental limit to the precision of a gravitational-wave detector [85], which provides an answer of how far we can push the detector sensitivity.

In Figure 4, we consider the variation of the optimal symmetrized noise spectral density $S_{\mathrm{FF}}(\omega)$ with the increase of the frequency $\omega$, analogously similar to the corresponding illustration in Ref. [41]. Here we set the OPA pump gain as $G / \kappa=0.2$. As illustrated in Figure 4(a), the SQL can be well suppressed at frequencies below the mechanical resonance, i.e., we can increase the force sensitivity by nearly two-orders of magnitude in the low-frequency domain. Note that recently, a quantum expander for gravitationalwave observatories has been demonstrated in Ref. [86], where quantum uncertainty can be squeezed even at high frequencies, while maintaining the low-frequency sensitivity unchanged, thus, expanding the detection bandwidth. In Figures 4(b)-4(c), we depict the two key parameters, i.e., the optomechanical coupling strength $g_{\text {opt }}$ and intracavity phase $\phi$, required for the minimum symmetrized noise spectral density, in the limits of different phases $\theta$ and frequencies $\omega$.

From what has been discussed above, it would be reasonable to overcome the SQL by tuning the OPA pump phase. However, because of this nonzero pump phase, the canonical position $\left(x_{a}\right)$ and momentum $\left(p_{a}\right)$ operators become correlated, causing a loss of quantum efficiency which cannot be ignored (see Appendix B for more details). For instance, the dashed line in Figure 2(b) illustrates the case of $\theta=\phi=0$, in which $x_{a}$ and $p_{a}$ are decoupled, circumventing quantum efficiency losses successfully, but a loss of mechanicalmode information is inevitable in the regions divided by the dashed line. Additionally, as the frequency increases, there is an unavoidable quantum efficiency loss shown in Figure 4. Nonetheless, in the specific regime where $\kappa \gg \omega$ and $\Delta=0$, we are able to achieve quantum noise reduction without losing mechanical-mode information. In the limit of $\kappa \gg \omega$, according to the coefficients given in Appendix C, we find:

$$
\mu_{ \pm} \lambda_{ \pm} \approx \frac{4 G \sin \theta \pm 2 g^{2} \chi_{m} \sin ^{2}\left(\phi+\phi_{ \pm}\right)}{\kappa \mp 4 G \cos \theta \pm g^{2} \chi_{m} \sin (2 \phi)},
$$

where $\phi_{ \pm}=(1 \pm 1) \pi / 4$. Hence, we obtain $\tan \phi=$ $\mu_{-} \lambda_{-}$and $\cot \phi \neq \mu_{+} \lambda_{+}$, i.e., $f_{+}=0$ and $f_{-} \neq$ 0 . Utilizing the input-output relations, the output canonical position quadrature $x_{a}^{\text {out }}$ does not contain $f_{\text {in }}$; thus, the detected squeezed quadrature carries all of the mechanical quantum information, and the SQL can be reached or surpassed, as shown in Figures 23 , whose parameters utilized in calculations obey the approximation $\kappa \gg \omega$. 

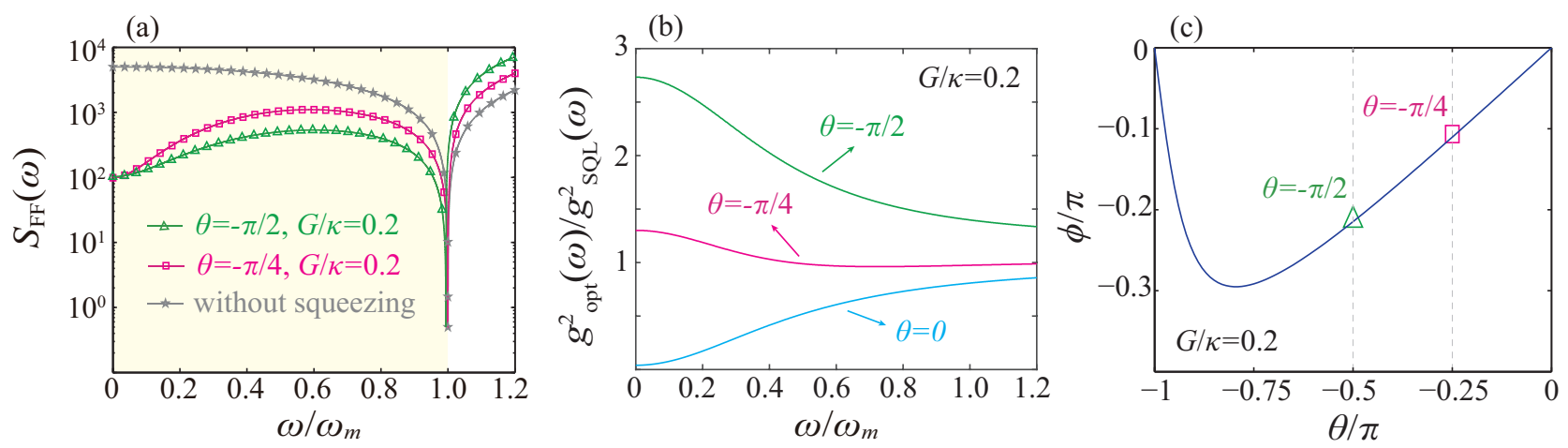

FIG. 4. (Color online) (a) Noise power spectral density $S_{\mathrm{FF}}(\omega)$ at the optimal power vs frequency $\omega$. The gray curve corresponds to $S_{\mathrm{SQL}}(\omega)$ without squeezing. (b) Optimal coupling strength as functions of the OPA pump phase and the detection frequency. (c) Intracavity phase with varying the OPA pump phase. The parametric gain is set as $G / \kappa=0.2$.

\section{CONCLUSION}

In conclusion, we have investigated weak-force sensing in a squeezed cavity and theoretically showed that (i) the SQL cannot be surpassed in the case of $G=0$ or $\theta=0$, (ii) the measurement precision of weak-force detection can be remarkably improved at the coupling strength smaller than $g_{\mathrm{SQL}}$ by tuning the parametric phase and gain, and (iii) under the approximation of $\kappa \gg \omega$, quantum noise can be reduced without losing mechanical-mode information. Our work provides new insight in strengthening the sensitivity of a force sensor with the assistance of intracavity squeezing, which can be also extended into other systems of quantum sensing with e.g., waveguide [84, 87], interferometer [88], or parity-time $(\mathcal{P} \mathcal{T})$ symmetric microcavity [89-91]. In the future, we plan to extend our work to study the weakforce measurement with the help of two-mode squeezing or quantum entanglement [92-94], squeezed mechanical modes [70, 95], or squeezed sources in hybrid COM devices [96, 97].

\section{ACKNOWLEDGMENTS}

This work was supported by the National Natural Science Foundation of China (NSFC) (Grants Nos. 11474087, and 11774086), the Key Program of NSFC (Grant No. 11935006), and the HuNU Program for Talented Youth.

\section{APPENDIX A: DERIVATION OF THE EFFECTIVE HAMILTONIAN}

In our system, the cavity with resonant frequency $\omega_{a}$ and damping rate $\kappa$, is driven by an input beam $a_{\text {in }}$ at frequency $\omega_{l}$. The left mirror is movable, which supports a mechanical mode with frequency $\omega_{m}$ and damping rate $\gamma_{m}$. An external force $F_{\text {ex }}$ is applied on the left-hand mirror and the cavity adjoins the movable mirror with coupling strength $g_{0}=x_{\mathrm{zpf}} \omega_{a} / L$, where $L$ is the length of the cavity. When a pump field at frequency $2 \omega_{l}$ interacts with a second-order nonlinear optical crystal, the output frequency becomes $\omega_{l}$ [40]. The nonlinear gain $G$ of the degenerate OPA with pump phase $\theta$ is proportional to the pump field. By transforming into a frame rotating at the incoming laser frequency $\omega_{l}$ with $\Delta_{a}=\omega_{a}-\omega_{l}$, Hamiltonian (1) can be rewritten as:

$$
H=-i \hbar U \frac{\mathrm{d} U^{\dagger}}{\mathrm{d} t}+U H_{0} U^{\dagger}
$$

via the unitary transformation $U(t)=\exp \left(i \omega_{l} a^{\dagger} a t\right)$. Using the relation

$$
e^{A} B e^{-A}=B+[A, B]+\frac{1}{2 !}[A,[A, B]]+\cdots,
$$

we obtain $[48,57]$ :

$$
\begin{aligned}
H= & \hbar \Delta_{a} a^{\dagger} a+\hbar \frac{\omega_{a}}{L} x a^{\dagger} a+\frac{p^{2}}{2 m}+\frac{1}{2} m \omega_{m}^{2} x^{2} \\
& +i \hbar G\left(e^{i \theta} a^{\dagger 2}-e^{-i \theta} a^{2}\right)
\end{aligned}
$$

where $m$ stands for the effective mass of the mechanical mode, while $x$ and $p$ represent the position and momentum operators, respectively.

In the Heisenberg picture, the dynamics of an operator $\mathcal{O}$ of a quantum system can be determined via the Heisenberg equation of motion:

$$
\dot{\mathcal{O}}(t)=\frac{1}{i \hbar}[\mathcal{O}(t), H(t)] .
$$

By introducing dissipation and noise terms, we have:

$$
\begin{aligned}
\dot{X} & =\omega_{m} P, \\
\dot{P} & =-\omega_{m} X-\gamma_{m} P-g_{0} a^{\dagger} a+\sqrt{2 \gamma_{m}}\left(f_{\mathrm{th}}+f_{\mathrm{ex}}\right), \\
\dot{a} & =-i\left(\Delta_{a}+g_{0} X\right) a+2 G e^{i \theta} a^{\dagger}-\frac{\kappa}{2} a+\sqrt{\kappa} a_{\mathrm{in}},
\end{aligned}
$$


where $\kappa$ and $\gamma_{m}$ are the decay rates of the optical cavity and the mechanical oscillator, respectively. $X=x / x_{\mathrm{zpf}}$ and $P=p / p_{\mathrm{zpf}}$ denote the dimensionless displacement and momentum operators of the mechanical mode, where $x_{\mathrm{zpf}}=\sqrt{\hbar /\left(m \omega_{m}\right)}$ and $p_{\mathrm{zpf}}=\sqrt{\hbar m \omega_{m}}$ are the zeropoint position and momentum fluctuations, respectively. The input noise operator $a_{\text {in }}(t)$ fulfills the correlation relations [41, 49]:

$$
\begin{aligned}
\left\langle a_{\text {in }}^{\dagger}(t) a_{\text {in }}\left(t^{\prime}\right)\right\rangle & =0, \\
\left\langle a_{\text {in }}(t) a_{\text {in }}^{\dagger}\left(t^{\prime}\right)\right\rangle & =\delta\left(t-t^{\prime}\right), \\
\left\langle a_{\text {in }}(t) a_{\text {in }}\left(t^{\prime}\right)\right\rangle & =\left\langle a_{\text {in }}^{\dagger}(t) a_{\text {in }}^{\dagger}\left(t^{\prime}\right)\right\rangle=0 .
\end{aligned}
$$

Moreover, $f_{\text {th }}$ and $f_{\text {ex }}$ represent the scaled thermal and external forces given, respectively, by:

$$
f_{\mathrm{th}}=\frac{\xi}{\sqrt{2 \hbar m \gamma_{m} \omega_{m}}}, \quad f_{\mathrm{ex}}=\frac{F_{\mathrm{ex}}}{\sqrt{2 \hbar m \gamma_{m} \omega_{m}}},
$$

where $\xi$ and $F_{\text {ex }}$ are the corresponding thermal and external forces, respectively.

When the mechanical resonator is in thermal equilibrium at environment temperature $T$, the Bose-Einstein statistics determines the occupancy probability $p(n)$ of each energy level, given by:

$$
p(n)=\delta^{n}(1-\delta),
$$

where $\delta=\exp \left[-\hbar \omega_{m} /\left(k_{B} T\right)\right]$. Therefore, the mean number $\bar{n}$ of phonons in thermal equilibrium is

$$
\bar{n}=\sum_{n=0}^{\infty} n p(n)=\left(\delta^{-1}-1\right)^{-1} .
$$

In the high temperature limit of $k_{B} T \gg \hbar \omega_{m}$, Eq. (A9) can be simplified to the familiar expression:

$$
\bar{n} \approx \frac{k_{B} T}{\hbar \omega_{m}},
$$

and according to Eq. (5), the thermal noise force satisfies the correlation function as follows [41]:

$$
\left\langle\xi(t) \xi\left(t^{\prime}\right)\right\rangle=2 m \gamma_{m} k_{B} T \delta\left(t-t^{\prime}\right) .
$$

Combining Eq. (A7) with Eq. (A11), we obtain:

$$
\left\langle f_{\mathrm{th}}(t) f_{\mathrm{th}}\left(t^{\prime}\right)\right\rangle=\frac{k_{B} T}{\hbar \omega_{m}} \delta\left(t-t^{\prime}\right) .
$$

Hence, Eq. (A12) becomes [41]:

$$
\left\langle f_{\text {th }}(t) f_{\text {th }}\left(t^{\prime}\right)\right\rangle=\bar{n} \delta\left(t-t^{\prime}\right) .
$$

\section{APPENDIX B: LINEAR RESPONSE}

To solve the nonlinear Heisenberg-Langevin equations in Eq. (3), we linearize the operators around the steadystate values $\left(\bar{X}, \bar{P}, \alpha, \bar{f}_{\text {th }}, \bar{f}_{\text {ex }}, \alpha_{\text {in }}\right)$, i.e., insert the ansatz

$$
\begin{aligned}
X & =\bar{X}+\delta X, & P & =\bar{P}+\delta P, \\
a & =\alpha+\delta a, & f_{\mathrm{th}} & =\bar{f}_{\mathrm{th}}+\delta f_{\mathrm{th}}, \\
f_{\mathrm{ex}} & =\bar{f}_{\mathrm{ex}}+\delta f_{\mathrm{ex}}, & a_{\mathrm{in}} & =\alpha_{\mathrm{in}}+\delta a_{\mathrm{in}},
\end{aligned}
$$

into Eq. (A5), and retain only the first-order terms, then we obtain:

$$
\begin{aligned}
\dot{X} & =\omega_{m} P, \\
\dot{P} & =-\omega_{m} X-\gamma_{m} P-g_{0}\left(\alpha^{*} a+\alpha a^{\dagger}\right)+\sqrt{2 \gamma_{m}} f_{\mathrm{in}}, \\
\dot{a} & =-\left(i \Delta_{a}+\frac{\kappa}{2}\right) a-i g_{0}(\bar{X} a+\alpha X)+2 G e^{i \theta} a^{\dagger}+\sqrt{\kappa} a_{\mathrm{in}} .
\end{aligned}
$$

where $f_{\text {in }}=f_{\text {th }}+f_{\text {ex }}$ and we consider the thermal and external noises average to 0 . For simplicity, we set the integral constants to zero.

When all time derivatives vanish, the steady-state values fulfill the self consistent equations:

$$
\begin{aligned}
& 0=\omega_{m} \bar{P}, \\
& 0=-\omega_{m} \bar{X}-\gamma_{m} \bar{P}-g_{0} \alpha^{*} \alpha, \\
& 0=-\left(i \Delta_{a}+\frac{\kappa}{2}\right) \alpha-i g_{0} \bar{X} \alpha+2 G e^{i \theta} \alpha^{*}+\sqrt{\kappa} \alpha_{\text {in }} .
\end{aligned}
$$

The steady-state solution of Eq. (B3) can be given by: $\bar{P}=0, \bar{X}=-g_{0} n_{a} / \omega_{m}$, and

$$
\alpha=\frac{\sqrt{\kappa}}{2 \sigma_{+}}\left[(\kappa-2 i \Delta) \alpha_{\mathrm{in}}+4 G e^{i \theta} \alpha_{\mathrm{in}}^{*}\right]=|\alpha| e^{i \phi},
$$

where $\sigma_{ \pm}=\kappa^{2} / 4 \pm \Delta^{2} \mp 4 G^{2}$ and $\phi$ is the phase of the intracavity field. We have defined the effective detuning $\left(\Delta=\Delta_{a}+g_{0} \bar{X}\right)$ and the mean intracavity photon number $\left(n_{a}=|\alpha|^{2}\right)$.

Since phase is relative while phase difference is absolute, we focus on the phase difference between the external and internal optical fields and choose the incoming field as the zero phase reference, i.e., $\alpha_{\text {in }}=$ $\left|\alpha_{\text {in }}\right|=\sqrt{P_{\text {in }} / \hbar \omega_{l}}$, where $P_{\text {in }}$ denotes the input laser power [17]. Thus, Eq. (B4) can be simplified to the following expression:

$$
\alpha=\frac{\sqrt{\kappa} \alpha_{\text {in }}}{2 \sigma_{+}}\left(\kappa-2 i \Delta+4 G e^{i \theta}\right)=|\alpha| e^{i \phi} .
$$

The assumption that $\alpha_{\text {in }}$ is real, makes the phase $\phi$ dependent on $\kappa, G, \Delta$, and $\theta$, as follows:

$$
\phi=\arctan \left(\frac{4 G \sin \theta-2 \Delta}{4 G \cos \theta+\kappa}\right) .
$$

Furthermore, $|\alpha|$ can be solved from Eq. (B5):

$$
|\alpha|=\left|\frac{\sqrt{\kappa} \alpha_{\mathrm{in}}}{\sigma_{+}}\right| \sqrt{\sigma+2 G(\kappa \cos \theta-2 \Delta \sin \theta)},
$$

where $\sigma=\kappa^{2} / 4+\Delta^{2}+4 G^{2}$. In the limit of $\Delta=0$, we use experimentally accessible parameters, which have 
been listed in Section II, to estimate the magnitude of $|\alpha|$ :

$$
|\alpha| \geq\left|\frac{2 \sqrt{\kappa} \alpha_{\text {in }}}{\kappa+4 G}\right| \sim 10^{2} \gg 1 .
$$

Hence, our calculations satisfy the strong-driving condition $|\alpha| \gg 1$ [40] and this linearized model has been proved valid and physically reasonable for our COM system.

To obtain the solutions of Eq. (B2), we define the quadratures of input/output fields as $x_{a}^{u}=$ $\left(a_{u}+a_{u}^{\dagger}\right) / \sqrt{2}$ and $p_{a}^{u}=\left(a_{u}-a_{u}^{\dagger}\right) /(\sqrt{2} i)$, where $u=$ in, out, analogously similar to the canonical position and momentum operators (given in Section II). Then, we have the linearized Heisenberg-Langevin equations given in Eq. (8), with the coefficients:

$$
\begin{aligned}
\mathrm{A} & =\left(\begin{array}{cccc}
0 & 0 & 0 & 0 \\
0 & \sqrt{2 \gamma_{m}} & 0 & 0 \\
0 & 0 & \sqrt{\kappa} & 0 \\
0 & 0 & 0 & \sqrt{\kappa}
\end{array}\right) \\
\mathrm{C} & =\left(\begin{array}{cccc}
0 & \omega_{m} & 0 & 0 \\
-\omega_{m} & -\gamma_{m} & -g \cos \phi & -g \sin \phi \\
g \sin \phi & 0 & C_{-} & S_{+} \\
-g \cos \phi & 0 & S_{-} & -C_{+}
\end{array}\right),
\end{aligned}
$$

where $C_{ \pm}=2 G \cos \theta \pm \kappa / 2, S_{ \pm}=2 G \sin \theta \pm \Delta$, and $g=\sqrt{2} g_{0}|\alpha|$ is the effective linearized optomechanical coupling strength. Using the Fourier transform of Eq. (8), we obtain the linearized Heisenberg-Langevin equations in the frequency domain:

$$
-i \omega \tilde{\mathrm{v}}=\mathrm{C} \tilde{\mathrm{v}}+\mathrm{A} \tilde{\mathrm{v}}_{\mathrm{in}},
$$

where $\tilde{\mathrm{v}} \equiv \mathrm{v}(\omega)=\left(\tilde{X}, \tilde{P}, \tilde{x}_{a}, \tilde{p}_{a}\right)^{\mathrm{T}}, \tilde{\mathrm{v}}_{\text {in }} \equiv \mathrm{v}_{\text {in }}(\omega)=$ $\left(0, \tilde{f}_{\text {in }}, \tilde{x}_{a}^{\text {in }}, \tilde{p}_{a}^{\text {in }}\right)^{\mathrm{T}}$, and $\tilde{f}_{\text {in }} \equiv \tilde{f}_{\text {in }}(\omega) ; \quad \tilde{o} \equiv o(\omega)$, $\tilde{s}_{a} \equiv s_{a}(\omega)$, and $\tilde{s}_{a}^{\text {in }} \equiv s_{a}^{\text {in }}(\omega)$, for $o=X, P$, and $s=x, p$. From Eqs. (8) and (B9), we can see when inserting a degenerate OPA medium into the Fabry-Pérot cavity, the canonical position $\left(x_{a}\right)$ and momentum $\left(p_{a}\right)$ operators are decoupled only if $S_{ \pm}=0$. Specifically, in the dashed line showed in Fig. 2(b), substituting $\Delta=\theta=0\left(S_{ \pm}=0\right)$ into Eq. (B10), we find that all the information is imprinted on the squeezed canonical momentum quadrature: $\tilde{x}_{a}=\rho_{-} \sqrt{\kappa} \tilde{x}_{a}^{\text {in }}$, and

$$
\begin{aligned}
\tilde{p}_{a}= & g^{2} \chi_{m} \rho_{+} \rho_{-} \sqrt{\kappa} \tilde{x}_{a}^{\text {in }}+\rho_{+} \sqrt{\kappa} p_{a}^{\text {in }} \\
& -g \chi_{m} \rho_{+} \sqrt{2 \gamma_{m}} \tilde{f}_{\text {in }},
\end{aligned}
$$

where $\rho_{ \pm}=\left(\chi^{-1} \pm 2 G\right)^{-1}$, and the susceptibilities of the cavity field and the mechanical oscillator are respectively defined as [49]:

$$
\begin{aligned}
\chi(\omega) & =(\kappa / 2-i \omega)^{-1}, \\
\chi_{m}(\omega) & =\omega_{m}\left(\omega_{m}^{2}-\omega^{2}-i \omega \gamma_{m}\right)^{-1} .
\end{aligned}
$$

Clearly, there are no correlations between this squeezed quadrature and its canonically conjugated quadrature $\tilde{x}_{a}$. However, on the both sides of the dividing line in Fig. 2(b), loss of mechanical-mode information inevitably exists, for the two quadratures are correlated. Therefore, we can conclude that under the resonance condition $\Delta=0$, quantum efficiency losses are dependent on the coupling of the quadratures $x_{a}$ and $p_{a}$ (i.e., the coefficients $S_{ \pm}$or the phase $\theta$ of the OPA pump). Our analysis of the stability conditions for the matrix $\mathrm{C}$, in Eq. (B9), and the input-output relations are given in Appendix C.

\section{APPENDIX C: STABILITY CONDITIONS AND INPUT-OUTPUT RELATIONS}

The system is stable only if all the eigenvalues $\lambda$ of the matrix $\mathrm{C}$ have negative real parts [98]. It is well known that the characteristic equation $|\mathrm{C}-\lambda \mathrm{I}|=0$ can be reduced to:

$$
\lambda^{4}+\mathrm{C}_{3} \lambda^{3}+\mathrm{C}_{2} \lambda^{2}+\mathrm{C}_{1} \lambda+\mathrm{C}_{0}=0 .
$$

Hence, we obtain the stability conditions of the system from the Routh-Hurwitz criterion [98]:

$$
\begin{aligned}
& 0<\mathrm{C}_{3}, \\
& 0<\mathrm{C}_{3} \mathrm{C}_{2}-\mathrm{C}_{1}, \\
& 0<\mathrm{C}_{3} \mathrm{C}_{2} \mathrm{C}_{1}-\left(\mathrm{C}_{1}^{2}+\mathrm{C}_{3}^{2} \mathrm{C}_{0}\right) .
\end{aligned}
$$

Specifically, all the external parameters should be chosen to satisfy the stability conditions in Eq. (C2), where the coefficients of the characteristic equation can be given by:

$$
\begin{aligned}
& \mathrm{C}_{3}=\kappa+\gamma_{m}, \\
& \mathrm{C}_{2}=\omega_{m}^{2}+\kappa \gamma_{m}+\sigma_{+}, \\
& \mathrm{C}_{1}=\kappa \omega_{m}^{2}+\sigma_{+} \gamma_{m}, \\
& \mathrm{C}_{0}=\sigma_{+} \omega_{m}^{2}+2 g^{2} G \omega_{m} \sin (2 \phi-\theta)-g^{2} \omega_{m} \Delta .
\end{aligned}
$$

By solving Eq. (B10), we obtain the quadratures $\tilde{x}_{a}$ and $\tilde{p}_{a}$ :

$$
\begin{aligned}
\tilde{x}_{a}= & g f_{+} \chi_{+} \chi_{m} \sqrt{2 \gamma_{m}} \tilde{f}_{\mathrm{in}}+\chi_{+} \sqrt{\kappa} \tilde{x}_{a}^{\text {in }} \\
& +\mu_{-} \lambda_{-} \chi_{+} \sqrt{\kappa} \tilde{p}_{a}^{\text {in }}, \\
\tilde{p}_{a}= & g f_{-} \chi_{-} \chi_{m} \sqrt{2 \gamma_{m}} \tilde{f}_{\text {in }}+\chi_{-} \sqrt{\kappa} \tilde{p}_{a}^{\text {in }} \\
& +\mu_{+} \lambda_{+} \chi_{-} \sqrt{\kappa} \tilde{x}_{a}^{\text {in }},
\end{aligned}
$$

where $\tilde{f}_{r} \equiv f_{r}(\omega), \tilde{s}_{a}^{u} \equiv s_{a}^{u}(\omega)$, for $s=x, p, u=$ in, out, and $r=$ in, th, ex, add. Moreover, the coefficients in Eq. (C4) are given by:

$$
\begin{aligned}
f_{-}(\omega) & =\mu_{+} \lambda_{+} \sin \phi-\cos \phi, \\
f_{+}(\omega) & =\sin \phi-\mu_{-} \lambda_{-} \cos \phi, \\
\chi_{ \pm}(\omega) & =\left(\lambda_{ \pm}^{-1}-\mu_{+} \mu_{-} \lambda_{\mp}\right)^{-1}, \\
\mu_{ \pm}(\omega) & =\mp \Delta+2 G \sin \theta \pm g^{2} \chi_{m} \cos ^{2} \phi, \\
\lambda_{ \pm}(\omega) & =\left[\chi^{-1} \mp 2 G \cos \theta \pm \frac{1}{2} g^{2} \chi_{m} \sin (2 \phi)\right]^{-1} .
\end{aligned}
$$


Using the input-output relations $\tilde{x}_{a}^{\text {out }}=\sqrt{\kappa} \tilde{x}_{a}-\tilde{x}_{a}^{\text {in }}$, $\tilde{p}_{a}^{\text {out }}=\sqrt{\kappa} \tilde{p}_{a}-\tilde{p}_{a}^{\text {in }}$, the quadratures of the output fields are given by:

$$
\begin{aligned}
\tilde{x}_{a}^{\text {out }}= & g f_{+} \chi_{+} \chi_{m} \sqrt{2 \kappa \gamma_{m}} \tilde{f}_{\text {in }}+\mu_{-} \lambda_{-} \chi_{+} \kappa \tilde{p}_{a}^{\text {in }} \\
& +\left(\chi_{+} \kappa-1\right) \tilde{x}_{a}^{\text {in }}, \\
\tilde{p}_{a}^{\text {out }}= & g f_{-} \chi_{-} \chi_{m} \sqrt{2 \kappa \gamma_{m}} \tilde{f}_{\text {in }}+\mu_{+} \lambda_{+} \chi_{-} \kappa \tilde{x}_{a}^{\text {in }} \\
& +\left(\chi_{-} \kappa-1\right) \tilde{p}_{a}^{\text {in }} .
\end{aligned}
$$

\section{APPENDIX D: MOTIONAL NOISE SPECTRUM}

Herein, we describe the outcoming optical field and the laser field with the annihilation operators $a_{\text {out }}(t)$ and $a_{\mathrm{LO}}(t)$. When they interact with a 50/50 beam splitter simultaneously, extracavity photons can be transformed into photoelectrons, generating two photocurrents:

$$
i_{k}(t)=n_{k}(t)=a_{k}^{\prime \dagger}(t) a_{k}^{\prime}(t),
$$

where $k=1,2$ and $n_{k}$ are the photon number operators measured in the two detectors. Making the parametric approximation for the photon number of the laser field, $\left\langle n_{\mathrm{LO}}\right\rangle \gg 1$, we have $a_{\mathrm{LO}} \approx \alpha_{\mathrm{LO}}$, where $\alpha_{\mathrm{LO}}$ denotes the amplitude of the local oscillator. In homodyne detection, the relations between $a_{\text {out }}, a_{\mathrm{LO}}$ and $a_{k}^{\prime}$ are expressed as:

$$
a_{k}^{\prime}=\frac{1}{\sqrt{2}}\left[a_{\mathrm{out}}+(-1)^{k} a_{\mathrm{LO}}\right] .
$$

We measure the difference of the intensities, which can be written as:

$$
\begin{aligned}
i_{\mathrm{det}} & =i_{2}-i_{1}=\alpha_{\mathrm{LO}}^{*} a_{\text {out }}+\alpha_{\mathrm{LO}} a_{\mathrm{out}}^{\dagger} \\
& =\left|\alpha_{\mathrm{LO}}\right|\left(e^{-i \varphi} a_{\mathrm{out}}+e^{i \varphi} a_{\mathrm{out}}^{\dagger}\right),
\end{aligned}
$$

where we have used $a_{\mathrm{LO}} \approx \alpha_{\mathrm{LO}}$, and $\varphi$ represents the phase of the local oscillator. Thus, the values of $\varphi$ and $\left|\alpha_{\mathrm{LO}}\right|$ can be arbitrary.

We define dimensionless quadrature operators $x_{a, \text { out }}^{\varphi}$ and $p_{a, \text { out }}^{\varphi}$ rotated by a phase angle $\varphi$ from $x_{a}^{\text {out }}$ and $p_{a}^{\text {out }}$ as follows:

$$
\left(\begin{array}{l}
x_{a, \text { out }}^{\varphi} \\
p_{a, \text { out }}^{\varphi}
\end{array}\right)=\left(\begin{array}{cc}
\cos \varphi & \sin \varphi \\
-\sin \varphi & \cos \varphi
\end{array}\right)\left(\begin{array}{l}
x_{a}^{\text {out }} \\
p_{a}^{\text {out }}
\end{array}\right),
$$

which obey the commutation relation $\left[x_{a, \text { out }}^{\varphi}, p_{a, \text { out }}^{\varphi}\right]=i$. Thus, we obtain the detected field operator:

$$
i_{\mathrm{det}}(t)=\sqrt{2}\left|\alpha_{\mathrm{LO}}\right| x_{a, \text { out }}^{\varphi} .
$$

We use $\psi$ to describe the phase of the outcoming field, and Eq. (B5) yields for the expression of $\psi$ via the input- output relation:

$$
\begin{aligned}
\alpha_{\text {out }} & =\left|\alpha_{\text {out }}\right| e^{i \psi}=\sqrt{\kappa} \alpha-\alpha_{\text {in }} \\
& =\frac{\kappa \alpha_{\text {in }}}{2 \sigma_{+}}\left(\kappa-2 i \Delta+4 G e^{i \theta}\right)-\alpha_{\text {in }} .
\end{aligned}
$$

For the assumption that $\alpha_{\text {in }}$ is real, $\psi$ is dependent on $\kappa$, $G, \Delta$, and $\theta$,

$$
\psi=\arctan \left(\frac{2 G \kappa \sin \theta-\Delta \kappa}{2 G \kappa \cos \theta+\sigma_{-}}\right),
$$

where $\sigma_{-}$has been defined below Eq. (B4).

In the specific case of an optomechanical system without detuning and OPA $(\Delta=G=0)$, we obtain $\phi=\psi=0$ from Eqs. (B6) and (D7), therefore, $\mu_{ \pm}, \lambda_{ \pm}$, and $\chi_{ \pm}$can be simplified to: $\mu_{+}=g^{2} \chi_{m}, \mu_{-}=0$, and $\lambda_{ \pm}=\chi_{ \pm}=\chi=(\kappa / 2-i \omega)^{-1}$. Thus, Eq. (C6) can be written as $\tilde{x}_{a}^{\text {out }}=\kappa_{+} \tilde{x}_{a}^{\text {in }} / \kappa_{-}$and

$$
\tilde{p}_{a}^{\text {out }}=\frac{g^{2} \chi_{m} \kappa}{\kappa_{-}^{2}} \tilde{x}_{a}^{\text {in }}+\frac{\kappa_{+}}{\kappa_{-}} \tilde{p}_{a}^{\text {in }}-\frac{g \chi_{m} \sqrt{2 \kappa \gamma_{m}}}{\kappa_{-}} \tilde{f}_{\text {in }} .
$$

where $\kappa_{ \pm}=\kappa / 2 \pm i \omega$. Apparently, there is no mechanical-mode information on the quadrature $\tilde{x}_{a}^{\text {out }}$ and we focus upon only the case where $\varphi=\pi / 2$, so that the total external force can be expressed as:

$$
\begin{aligned}
F & =\frac{1}{F_{f}(\omega)} x_{a, \text { out }}^{\pi / 2}=\frac{1}{F_{f}(\omega)} \tilde{p}_{a}^{\text {out }}=\tilde{f}_{\mathrm{ex}}+\tilde{f}_{\mathrm{add}} \\
& =\tilde{f}_{\mathrm{th}}+X_{a}(\omega) \tilde{x}_{a}^{\text {in }}+P_{a}(\omega) \tilde{p}_{a}^{\text {in }},
\end{aligned}
$$

where $X_{a}(\omega), P_{a}(\omega)$, and $F_{f}(\omega)$ have been defined in Eq. (12). Then, we have obtained the induced force:

$$
f_{\text {add }}(\omega)=\tilde{f}_{\mathrm{th}}+X_{a}(\omega) \tilde{x}_{a}^{\text {in }}+P_{a}(\omega) \tilde{p}_{a}^{\text {in }} .
$$

Moreover, we find that $X_{a}^{*}(\omega)=X_{a}(-\omega), P_{a}^{*}(\omega)=$ $P_{a}(-\omega)$.

The sensitivity of force measurement is commonly characterized via the noise power spectral density $S_{\mathrm{F}}$, which is given by [25]:

$$
S_{\mathrm{F}}(\omega)=\int \mathrm{d} \omega^{\prime}\left\langle f_{\text {add }}(\omega) f_{\text {add }}\left(\omega^{\prime}\right)\right\rangle .
$$

Utilizing the correlation functions of the input vacuum noise [25]:

$$
\begin{aligned}
& \left\langle x_{a}^{\mathrm{in}}(\omega) x_{a}^{\mathrm{in}}\left(\omega^{\prime}\right)\right\rangle=\left\langle p_{a}^{\mathrm{in}}(\omega) p_{a}^{\mathrm{in}}\left(\omega^{\prime}\right)\right\rangle=\frac{1}{2} \delta\left(\omega+\omega^{\prime}\right), \\
& \left\langle x_{a}^{\mathrm{in}}(\omega) p_{a}^{\mathrm{in}}\left(\omega^{\prime}\right)\right\rangle=-\left\langle p_{a}^{\mathrm{in}}(\omega) x_{a}^{\mathrm{in}}\left(\omega^{\prime}\right)\right\rangle=\frac{i}{2} \delta\left(\omega+\omega^{\prime}\right),
\end{aligned}
$$

we obtain the symmetrized noise spectral density $S_{\mathrm{FF}}$ given in Eq. (13). 
[1] A. A. Clerk, M. H. Devoret, S. M. Girvin, F. Marquardt, and R. J. Schoelkopf, "Introduction to quantum noise, measurement, and amplification," Rev. Mod. Phys. 82, 1155-1208 (2010).

[2] M. Aspelmeyer, T. J. Kippenberg, and F. Marquardt, "Cavity optomechanics," Rev. Mod. Phys. 86, 1391-1452 (2014).

[3] S.-W. Bin, X.-Y. Lü, T.-S. Yin, G.-L. Zhu, Q. Bin, and $\mathrm{Y}$. Wu, "Mass sensing by quantum criticality," Opt. Lett. 44, 630-633 (2019).

[4] S. Liu, B. Liu, Ju. Wang, T. Sun, and W.-X. Yang, "Realization of a highly sensitive mass sensor in a quadratically coupled optomechanical system," Phys. Rev. A 99, 033822 (2019).

[5] A. G. Krause, M. Winger, T. D. Blasius, Q. Lin, and O. Painter, "A high-resolution microchip optomechanical accelerometer," Nat. Photonics 6, 768 (2012).

[6] S. Qvarfort, A. Serafini, P. F. Barker, and S. Bose, "Gravimetry through non-linear optomechanics," Nat. Commun. 9, 3690 (2018).

[7] D. J. Wilson, V. Sudhir, N. Piro, R. Schilling, A. Ghadimi, and T. J. Kippenberg, "Measurementbased control of a mechanical oscillator at its thermal decoherence rate," Nature (London) 524, 325 (2015).

[8] M. Rossi, D. Mason, J. Chen, Y. Tsaturyan, and A. Schliesser, "Measurement-based quantum control of mechanical motion," Nature (London) 563, 53 (2018).

[9] N. Matsumoto, S. B. Cataño-Lopez, M. Sugawara, S. Suzuki, N. Abe, K. Komori, Y. Michimura, Y. Aso, and K. Edamatsu, "Demonstration of Displacement Sensing of a mg-Scale Pendulum for mm- and mg-Scale Gravity Measurements," Phys. Rev. Lett. 122, 071101 (2019).

[10] D. Mason, J. Chen, M. Rossi, Y. Tsaturyan, and A. Schliesser, "Continuous force and displacement measurement below the standard quantum limit," Nat. Phys. 15, 745 (2019).

[11] C. M. Caves, K. S. Thorne, R. W. P. Drever, V. D. Sandberg, and M. Zimmermann, "On the measurement of a weak classical force coupled to a quantum-mechanical oscillator. I. Issues of principle," Rev. Mod. Phys. 52, 341-392 (1980).

[12] S. Schreppler, N. Spethmann, N. Brahms, T. Botter, M. Barrios, and D. M. Stamper-Kurn, "Optically measuring force near the standard quantum limit," Science 344, 1486-1489 (2014).

[13] Y.-H. Zhou, Q.-S. Tan, X.-M. Fang, J.-F. Huang, and J.-Q. Liao, "Spectrometric detection of weak forces in cavity optomechanics," arXiv:1812.06752.

[14] A. Motazedifard, A. Dalafi, F. Bemani, and M. H. Naderi, "Force sensing in hybrid Bose-Einsteincondensate optomechanics based on parametric amplification," Phys. Rev. A 100, 023815 (2019).

[15] S. Basiri-Esfahani, A. Armin, S. Forstner, and W. P. Bowen, "Precision ultrasound sensing on a chip," Nat. Commun. 10, 132 (2019).

[16] T. Gebremariam, Y.-X. Zeng, M. Mazaheri, and C. Li, "Enhancing optomechanical force sensing via precooling and quantum noise cancellation," Sci. China-Phys. Mech. Astron. 63, 210311 (2020).
[17] W. P Bowen and G. J. Milburn, Quantum Optomechanics (CRC Press, Boca Raton, FL, 2015).

[18] V. B. Braginskiı and Y. I. Vorontsov, "Quantummechanical limitations in macroscopic experiments and modern experimental technique," Sov. Phys. Usp. 17, 644-650 (1975).

[19] K. S. Thorne, R. W. P. Drever, C. M. Caves, M. Zimmermann, and V. D. Sandberg, "Quantum Nondemolition Measurements of Harmonic Oscillators," Phys. Rev. Lett. 40, 667-671 (1978).

[20] V. B. Braginsky, Y. I. Vorontsov, and K. S. Thorne, "Quantum Nondemolition Measurements," Science 209, 547-557 (1980).

[21] Y. Ma, H. Miao, B. H. Pang, M. Evans, C. Zhao, J. Harms, R. Schnabel, and Y. Chen, "Proposal for gravitational-wave detection beyond the standard quantum limit through EPR entanglement," Nat. Phys. 13, 776 (2017).

[22] Y. Li, L. Pezzè, W. Li, and A. Smerzi, "Sensitivity bounds for interferometry with Ising Hamiltonians," Phys. Rev. A 99, 022324 (2019).

[23] S. Carrasco and M. Orszag, "Fisher Information, Weak Values and Correlated Noise in Interferometry," arXiv:1902.09247.

[24] C. M. Caves, "Quantum-mechanical noise in an interferometer," Phys. Rev. D 23, 1693-1708 (1981).

[25] X. Xu and J. M. Taylor, "Squeezing in a coupled twomode optomechanical system for force sensing below the standard quantum limit," Phys. Rev. A 90, 043848 (2014).

[26] A. Motazedifard, F. Bemani, M. H. Naderi, R. Roknizadeh, and D. Vitali, "Force sensing based on coherent quantum noise cancellation in a hybrid optomechanical cavity with squeezed-vacuum injection," New J. Phys. 18, 073040 (2016).

[27] J. B. Clark, F. Lecocq, R. W. Simmonds, J. Aumentado, and J. D. Teufel, "Observation of strong radiation pressure forces from squeezed light on a mechanical oscillator," Nat. Phys. 12, 683 (2016).

[28] N. S. Kampel, R. W. Peterson, R. Fischer, P.-L. Yu, K. Cicak, R. W. Simmonds, K. W. Lehnert, and C. A. Regal, "Improving Broadband Displacement Detection with Quantum Correlations," Phys. Rev. X 7, 021008 (2017).

[29] V. Sudhir, R. Schilling, S. A. Fedorov, H. Schütz, D. J. Wilson, and T. J. Kippenberg, "Quantum Correlations of Light from a Room-Temperature Mechanical Oscillator," Phys. Rev. X 7, 031055 (2017).

[30] C. B. Møller, R. A. Thomas, G. Vasilakis, E. Zeuthen, Y. Tsaturyan, M. Balabas, K. Jensen, A. Schliesser, K. Hammerer, and E. S. Polzik, "Quantum backaction-evading measurement of motion in a negative mass reference frame," Nature (London) 547, 191 (2017).

[31] A. Otterpohl, F. Sedlmeir, U. Vogl, T. Dirmeier, G. Shafiee, G. Schunk, D. V. Strekalov, H. G. L. Schwefel, T. Gehring, U. L. Andersen, G. Leuchs, and C. Marquardt, "Squeezed vacuum states from a whispering gallery mode resonator," arXiv:1905.07955.

[32] E. H. Kennard, "Zur Quantenmechanik einfacher Bewegungstypen," Z. Phys. 44, 326-352 (1927). 
[33] J. N. Hollenhorst, "Quantum limits on resonant-mass gravitational-radiation detectors," Phys. Rev. D 19, 1669 (1979).

[34] V. V. Dodonov, V. I. Man'ko, and V. N. Rudenko, "Nondemolition measurements in gravitational-wave experiments," Sov. Phys. JETP 51, 443-450 (1980).

[35] M. Xiao, L.-A. Wu, and H. J. Kimble, "Precision measurement beyond the shot-noise limit," Phys. Rev. Lett. 59, 278-281 (1987).

[36] LIGO Scientific Collaboration, "Enhanced sensitivity of the LIGO gravitational wave detector by using squeezed states of light," Nat. Photonics 7, 613 (2013).

[37] U. B. Hoff, G. I. Harris, L. S. Madsen, H. Kerdoncuff, M. Lassen, B. M. Nielsen, W. P. Bowen, and U. L. Andersen, "Quantum-enhanced micromechanical displacement sensitivity," Opt. Lett. 38, 1413-1415 (2013).

[38] M. J. Yap, J. Cripe, G. L. Mansell, T. G. McRae, R. L. Ward, B. J. J. Slagmolen, D. A. Shaddock, P. Heu, D. Follman, G. D. Cole, D. E. McClelland, and T. Corbitt, "Broadband reduction of quantum radiation pressure noise via squeezed light injection," arXiv:1812.09804.

[39] V. Peano, H. G. L. Schwefel, C. Marquardt, and F. Marquardt, "Intracavity Squeezing Can Enhance Quantum-Limited Optomechanical Position Detection through Deamplification," Phys. Rev. Lett. 115, 243603 (2015).

[40] S. Huang and G. S. Agarwal, "Robust force sensing for a free particle in a dissipative optomechanical system with a parametric amplifier," Phys. Rev. A 95, 023844 (2017).

[41] M. H. Wimmer, D. Steinmeyer, K. Hammerer, and M. Heurs, "Coherent cancellation of backaction noise in optomechanical force measurements," Phys. Rev. A 89, 053836 (2014).

[42] R. Huang, A. Miranowicz, J.-Q. Liao, F. Nori, and H. Jing, "Nonreciprocal Photon Blockade," Phys. Rev. Lett. 121, 153601 (2018).

[43] B. Li, R. Huang, X. Xu, A. Miranowicz, and H. Jing, "Nonreciprocal unconventional photon blockade in a spinning optomechanical system," Photon. Res. 7, 630641 (2019).

[44] M. A. Taylor, J. Janousek, V. Daria, J. Knittel, B. Hage, H.-A. Bachor, and W. P. Bowen, "Biological measurement beyond the quantum limit," Nat. Photonics 7, 229 (2013).

[45] S. Barzanjeh, S. Guha, C. Weedbrook, D. Vitali, J. H. Shapiro, and S. Pirandola, "Microwave Quantum Illumination," Phys. Rev. Lett. 114, 080503 (2015).

[46] L.-A. Wu, H. J. Kimble, J. L. Hall, and H. Wu, "Generation of Squeezed States by Parametric Down Conversion," Phys. Rev. Lett. 57, 2520-2523 (1986).

[47] P. Grangier, R. E. Slusher, B. Yurke, and A. LaPorta, "Squeezed-light-enhanced polarization interferometer," Phys. Rev. Lett. 59, 2153-2156 (1987).

[48] S. Huang and G. S. Agarwal, "Enhancement of cavity cooling of a micromechanical mirror using parametric interactions," Phys. Rev. A 79, 013821 (2009).

[49] S. Huang and A. Chen, "Improving the cooling of a mechanical oscillator in a dissipative optomechanical system with an optical parametric amplifier," Phys. Rev. A 98, 063818 (2018).

[50] H.-K. Lau and A. A. Clerk, "Ground state cooling and high-fidelity quantum transduction via parametrically- driven bad-cavity optomechanics," arXiv:1904.12984.

[51] M. Asjad, N. E. Abari, S. Zippilli, and D. Vitali, "Optomechanical cooling with intracavity squeezed light," arXiv:1906.00837.

[52] X.-Y. Lü, Y. Wu, J. R. Johansson, H. Jing, J. Zhang, and F. Nori, "Squeezed Optomechanics with Phase-Matched Amplification and Dissipation," Phys. Rev. Lett. 114, 093602 (2015).

[53] G. S. Agarwal and S. Huang, "Strong mechanical squeezing and its detection," Phys. Rev. A 93, 043844 (2016).

[54] W.-j. Gu, Z. Yi, L.-h. Sun, and Y. Yan, "Enhanced quadratic nonlinearity with parametric amplifications," J. Opt. Soc. Am. B 35, 652-657 (2018).

[55] W. Qin, A. Miranowicz, P.-B. Li, X.-Y. Lü, J. Q. You, and F. Nori, "Exponentially Enhanced LightMatter Interaction, Cooperativities, and Steady-State Entanglement Using Parametric Amplification," Phys. Rev. Lett. 120, 093601 (2018).

[56] C. Leroux, L. C. G. Govia, and A. A. Clerk, "Enhancing Cavity Quantum Electrodynamics via Antisqueezing: Synthetic Ultrastrong Coupling," Phys. Rev. Lett. 120, 093602 (2018).

[57] S. Huang and G. S. Agarwal, "Normal-mode splitting in a coupled system of a nanomechanical oscillator and a parametric amplifier cavity," Phys. Rev. A 80, 033807 (2009).

[58] V. B. Braginskiŭ and A. B. Manukin, "Ponderomotive effects of electromagnetic radiation," Sov. Phys. JETP 25, 653-655 (1967).

[59] R. Schnabel, "Squeezed states of light and their applications in laser interferometers," Phys. Rep. 684, 1-51 (2017).

[60] T. Corbitt, Y. Chen, F. Khalili, D. Ottaway, S. Vyatchanin, S. Whitcomb, and N. Mavalvala, "Squeezedstate source using radiation-pressure-induced rigidity," Phys. Rev. A 73, 023801 (2006).

[61] X. Zhang, Q.-T. Cao, Z. Wang, Y.-X. Liu, C.-W. Qiu, L. Yang, Q. Gong, and Y.-F. Xiao, "Symmetry-breakinginduced nonlinear optics at a microcavity surface," Nat. Photonics 13, 21-24 (2018).

[62] M. J. Collett and C. W. Gardiner, "Squeezing of intracavity and traveling-wave light fields produced in parametric amplification," Phys. Rev. A 30, 1386-1391 (1984).

[63] R. S. Bondurant, "Reduction of radiation-pressureinduced fluctuations in interferometric gravity-wave detectors," Phys. Rev. A 34, 3927-3931 (1986).

[64] T.-S. Yin, X.-Y. Lü, L.-L. Wan, S.-W. Bin, and Y. Wu, "Enhanced photon-phonon cross-Kerr nonlinearity with two-photon driving," Opt. Lett. 43, 2050-2053 (2018).

[65] A. Xuereb, R. Schnabel, and K. Hammerer, "Dissipative Optomechanics in a Michelson-Sagnac Interferometer," Phys. Rev. Lett. 107, 213604 (2011).

[66] A. Kronwald, F. Marquardt, and A. A. Clerk, "Dissipative optomechanical squeezing of light," New J. Phys. 16, 063058 (2014).

[67] C.-H. Bai, D.-Y. Wang, S. Zhang, and H.-F. Wang, "Qubit-assisted squeezing of mirror motion in a dissipative cavity optomechanical system," Sci. ChinaPhys. Mech. Astron. 62, 970311 (2019).

[68] J.-M. Pirkkalainen, E. Damskägg, M. Brandt, F. Massel, and M. A. Sillanpää, "Squeezing of Quantum Noise of Motion in a Micromechanical Resonator," Phys. Rev. 
Lett. 115, 243601 (2015).

[69] C. F. Ockeloen-Korppi, E. Damskägg, J.-M. Pirkkalainen, T. T. Heikkilä, F. Massel, and M. A. Sillanpää, "Noiseless Quantum Measurement and Squeezing of Microwave Fields Utilizing Mechanical Vibrations," Phys. Rev. Lett. 118, 103601 (2017).

[70] S. C. Burd, R. Srinivas, J. J. Bollinger, A. C. Wilson, D. J. Wineland, D. Leibfried, D. H. Slichter, and D. T. C. Allcock, "Quantum amplification of mechanical oscillator motion," Science 364, 1163-1165 (2019).

[71] W. Qin, A. Miranowicz, G. Long, J. Q. You, and F. Nori, "Proposal to test quantum wave-particle superposition on massive mechanical resonators," npj Quantum Inf. 5, 58 (2019).

[72] V. Giovannetti and D. Vitali, "Phase-noise measurement in a cavity with a movable mirror undergoing quantum Brownian motion," Phys. Rev. A 63, 023812 (2001).

[73] A. H. Safavi-Naeini, S. Gröblacher, J. T. Hill, J. Chan, M. Aspelmeyer, and O. Painter, "Squeezed light from a silicon micromechanical resonator," Nature (London) 500, 185 (2013).

[74] I. Shomroni, L. Qiu, D. Malz, A. Nunnenkamp, and T. J. Kippenberg, "Optical backaction-evading measurement of a mechanical oscillator," Nat. Commun. 10, 2086 (2019).

[75] U. Leonhardt, Measuring the Quantum State of Light (Cambridge University Press, Cambridge, 1997).

[76] A. Miranowicz, M. Paprzycka, A. Pathak, and F. Nori, "Phase-space interference of states optically truncated by quantum scissors: Generation of distinct superpositions of qudit coherent states by displacement of vacuum," Phys. Rev. A 89, 033812 (2014).

[77] J. Cripe, N. Aggarwal, R. Lanza, A. Libson, R. Singh, P. Heu, D. Follman, G. D. Cole, N. Mavalvala, and T. Corbitt, "Measurement of quantum back action in the audio band at room temperature," Nature (London) 568, 364 (2019).

[78] O. Arcizet, T. Briant, A. Heidmann, and M. Pinard, "Beating quantum limits in an optomechanical sensor by cavity detuning," Phys. Rev. A 73, 033819 (2006).

[79] M. Tsang and C. M. Caves, "Coherent Quantum-Noise Cancellation for Optomechanical Sensors," Phys. Rev. Lett. 105, 123601 (2010).

[80] M. Tsang and C. M. Caves, "Evading Quantum Mechanics: Engineering a Classical Subsystem within a Quantum Environment," Phys. Rev. X 2, 031016 (2012).

[81] C. F. Ockeloen-Korppi, E. Damskägg, J.-M. Pirkkalainen, A. A. Clerk, M. J. Woolley, and M. A. Sillanpää, "Quantum Backaction Evading Measurement of Collective Mechanical Modes," Phys. Rev. Lett. 117, 140401 (2016).

[82] V. Macrí, F. Nori, S. Savasta, and D. Zueco, "Optimal spin squeezing in cavity QED based systems," arXiv:1902.10377.

[83] H. Jing, H. Lü, S. K. Özdemir, T. Carmon, and F. Nori, "Nanoparticle sensing with a spinning resonator," Optica 5, 1424-1430 (2018).
[84] N. P. Mauranyapin, L. S. Madsen, L. Booth, L. Peng, S. C. Warren-Smith, E. P. Schartner, H. EbendorffHeidepriem, and W. P. Bowen, "Quantum noise limited nanoparticle detection with exposed-core fiber," Opt. Express 27, 18601-18611 (2019).

[85] H. Miao, N. D. Smith, and M. Evans, "Quantum Limit for Laser Interferometric Gravitational-Wave Detectors from Optical Dissipation," Phys. Rev. X 9, 011053 (2019).

[86] M. Korobko, Y. Ma, Y. Chen, and R. Schnabel, "Quantum expander for gravitational-wave observatories," arXiv:1903.05930.

[87] E. J. Allen, G. Ferranti, K. R. Rusimova, R. J. A. Francis-Jones, M. Azini, D. H. Mahler, T. C. Ralph, P. J. Mosley, and J. C. F. Matthews, "Passive, broadband and low-frequency suppression of laser amplitude noise to the shot-noise limit using hollow-core fibre," arXiv:1903.12598.

[88] B. Ouyang, Y. Li, M. Kruidhof, R. Horsten, K. W. A. V. Dongen, and J. Caro, "On-chip silicon MachZehnder interferometer sensor for ultrasound detection," Opt. Lett. 44, 1928-1931 (2019).

[89] Z.-P. Liu, J. Zhang, Ş. K. Özdemir, B. Peng, H. Jing, X.-Y. Lü, C.-W. Li, L. Yang, F. Nori, and Y.-x. Liu, "Metrology with $\mathcal{P} \mathcal{T}$-Symmetric Cavities: Enhanced Sensitivity near the $\mathcal{P} \mathcal{T}$-Phase Transition," Phys. Rev. Lett. 117, 110802 (2016).

[90] Ş. K. Özdemir, S. Rotter, F. Nori, and L. Yang, "Parity-time symmetry and exceptional points in photonics," Nat. Mater. 18, 783-798 (2019).

[91] Q. Zhong, J. Ren, M. Khajavikhan, D. N. Christodoulides, Ş. K. Özdemir, and R. El-Ganainy, "Sensing with Exceptional Surfaces in Order to Combine Sensitivity with Robustness," Phys. Rev. Lett. 122, 153902 (2019).

[92] T. A. Palomaki, J. D. Teufel, R. W. Simmonds, and K. W. Lehnert, "Entangling Mechanical Motion with Microwave Fields," Science 342, 710-713 (2013).

[93] Y. Takeuchi, Y. Matsuzaki, K. Miyanishi, T. Sugiyama, and W. J. Munro, "Quantum remote sensing with asymmetric information gain," Phys. Rev. A 99, 022325 (2019).

[94] S. Barzanjeh, E. S. Redchenko, M. Peruzzo, M. Wulf, D. P. Lewis, G. Arnold, and J. M. Fink, "Stationary entangled radiation from micromechanical motion," Nature (London) 570, 480-483 (2019).

[95] J. Suh, A. J. Weinstein, C. U. Lei, E. E. Wollman, S. K. Steinke, P. Meystre, A. A. Clerk, and K. C. Schwab, "Mechanically detecting and avoiding the quantum fluctuations of a microwave field," Science 344, 12621265 (2014).

[96] J. Li, S.-Y. Zhu, and G. S. Agarwal, "Squeezed states of magnons and phonons in cavity magnomechanics," Phys. Rev. A 99, 021801 (2019).

[97] C. Xu, L. Zhang, S. Huang, T. Ma, F. Liu, H. Yonezawa, Y. Zhang, and M. Xiao, "Sensing and tracking enhanced by quantum squeezing," Photon. Res. 7, A14-A26 (2019).

[98] A. Jeffrey and D. Zwillinger, Table of Integrals, Series, and Products, 6th ed. (Academic, New York, 2000). 